\title{
Cul4B is a novel prognostic marker in cholangiocarcinoma
}

\author{
PENGYU LI $^{1 *}$, LILI ZHANG $^{2 *}$, MUYI YANG $^{2}$, MEI QI $^{2}$, XING JIN $^{3}$ and BO HAN ${ }^{2,4}$ \\ ${ }^{1}$ Department of Emergency Surgery, Shandong University Qilu Hospital; ${ }^{2}$ Department of Pathology, \\ Shandong University Medical School; ${ }^{3}$ Department of Vascular Surgery, Shandong Provincial Hospital; \\ ${ }^{4}$ Department of Pathology, Shandong University Qilu Hospital, Jinan, Shandong 250012, P.R. China
}

Received July 26, 2015; Accepted October 27, 2016

DOI: $10.3892 / 01.2017 .6297$

\begin{abstract}
Cullin 4B (Cu14B), a scaffold protein that assembles the ubiquitin ligase complex, is involved in a wide variety of physiological and developmental processes, such as cell cycle progression, DNA damage response and gene expression regulation. Cul4B is overexpressed in various solid tumors. However, the prognostic value and role of Cul4B in cholangiocarcinoma (CCA) is largely unknown. The present study demonstrated that Cul4B was overexpressed in $21(26.6 \%)$ of 79 patients with intrahepatic CCA, and in 40 (28.6\%) of 140 patients with extrahepatic CCA (EHCC). Kaplan-Meier survival analysis suggested that $\mathrm{Cul} 4 \mathrm{~B}$ expression is an unfavorable prognostic factor in EHCC patients. Notably, Cul4B and epidermal growth factor receptor expression define a subset of CCA patients with poor prognosis. In vitro data indicated that $\mathrm{Cul} 4 \mathrm{~B}$ promotes the proliferation, migration and invasion of CCA cells. Furthermore, Cul4B expression promotes the epithelial-mesenchymal transition (EMT) process in CCA cells. Finally, Cul4B repressed the expression of the tumor suppressor genes P16 and phosphatase and tensin homolog. Collectively, the results of the present study revealed an important role of Cul4B in CCA with respect to initiating EMT. Cul4B expression may serve as a prognostic marker for patients with EHCC.
\end{abstract}

\section{Introduction}

Cholangiocarcinoma (CCA), anatomically classified into intrahepatic CCA (IHCC) and extrahepatic CCA (EHCC), is a highly aggressive malignancy due to early invasion, widespread metastasis and a lack of effective therapeutic approaches (1-3). Complete resection is the only way to

Correspondence to: Dr Bo Han, Department of Pathology, Shandong University Qilu Hospital, 107 Wenhua Xi Road, Jinan, Shandong 250012, P.R. China

E-mail: boh@sdu.edu.cn

*Contributed equally

Key words: cullin 4B, epidermal growth factor receptor, epithelial-mesenchymal transition, cholangiocarcinoma, prognosis cure the disease at present, but the recurrence rates of the patients that receive this treatment are high (4). Clinically, the prognostic factors of CCA have not been well established to date. Certain molecular biomarkers have been reported to be associated with poor survival and tumor progression, mucin (MUC)1, MUC4, fascin and epidermal growth factor receptor (EGFR), but the majority of biomarkers are not used routinely in clinical practice (5). Therefore, novel biomarkers for prognostic stratification and individualized therapy are required.

Cullin (Cul)-really interesting new gene ubiquitin ligase (CRL) complexes represent the largest known class of ubiquitin ligases, and are involved in a wide variety of physiological and developmental process, such as cell cycle progression, DNA damage response and gene expression regulation (6-8). In mammals, there are two Cul4 proteins, Cul4A and Cul4B, which share $82 \%$ identity in protein sequences. Notably, Cul4B, as opposed to $\mathrm{Cul} 4 \mathrm{~A}$, exhibits a nuclear localization signal, in its $\mathrm{N}$ terminus, and is localized in the nucleus, suggesting that Cul4B may be involved in nucleus-based functions (7). Previously, the present and other authors have reported that $\mathrm{Cul} 4 \mathrm{~B}$ is overexpressed in numerous solid tumor, types, such as lung, colon and liver cancer (9-11). Hu et al (11) suggested that Cul4B exerts an oncogenic effect by contributing to the epigenetic silencing of tumor suppressors. However, the expression and role of Cul4Bin the progression of CCA remain unknown.

Epithelial-mesenchymal transition (EMT), which involves the downregulation of epithelial markers and the upregulation of mesenchymal markers, has been shown to participate in the progression and metastasis of multiple types of cancer (12-14). However, the link between Cul4B and the EMT process is unclear in CCA.

The present study demonstrated that Cul4B is overexpressed in CCA, which may serve as an unfavorable prognostic factor in patients with EHCC, but not in patients with IHCC. The present study demonstrated that $\mathrm{Cul} 4 \mathrm{~B}$ expression is oncogenic and may promote the EMT process in CCA cells. $\mathrm{Cul}$ B+/EGFR+ defines a subset of EHCC patients with poor prognosis.

\section{Patients and methods}

Patients and tissue microarray (TMA) construction. The present study consisted of 219 CCA patients, 79 with IHCC and 140 with EHCC, who were treated between January 2007 and December 2013 at the Qilu Hospital of Shandong 
University (Jinan, China), Shandong Provincial Hospital (Jinan, China) and Affiliated Hospital of Qingdao University (Qingdao, China). Informed written consent was obtained from the patients. The present study was approved by the Institutional Review Board at the School of Medicine of Shandong University (Jinan, China). Patients that exhibited other types of malignancy or had succumbed to illness within 1 month subsequent to surgery were excluded from the study. Follow-up data were available for 194 patients, ranging between 4 and 92 months subsequent to the surgery (mean, 27 months). A total of three TMAs were constructed. Two cores of $1.0 \mathrm{~mm}$ in diameter were obtained from each representative tumor focus, and their morphology was confirmed by two pathologists. Detailed clinical and pathological profiles were obtained from the medical records of the patients and maintained in a secure relational database with TMA data. Patient demographics are shown in Table I.

Immunohistochemistry (IHC). IHC was performed as previously described (15). IHC staining was performed on TMA slides using the standardized labeled streptavidin biotin kit (Dako North America, Inc., Carpinteria, CA, USA) according to the protocol of the manufacturer. The 4- $\mu \mathrm{m}$ slides were then deparaffinized in xylene and rehydrated through graded ethanol and in deionized water in the final wash. The sections were submerged in an antigenic retrieval buffer (citric acid, $\mathrm{pH}$ 6.0) for heat-mediated retrieval by high pressure for $3 \mathrm{~min}$. The slides were then incubated overnight at $4^{\circ} \mathrm{C}$ with anti-Cul4B primary antibody (cat. no. c9995; 1:500 dilution; Sigma-Aldrich; Merck KGaA, Darmstadt, Germany). The slides were blindly evaluated by two independent pathologists. A previously described scoring system was utilized to validate Cul4B expression (15). The scores of two parameters were multiplied by the staining intensity $(0-3)$ and the percentage of positive cells, which ranged between 0 and 4; $(0,0-10$; $1,11-25 ; 2,26-50 ; 3,51-75$; and 4, 76-100\%). Final scores of $\geq 8$ were classified as overexpressed, while slides with scores $<8$ classified as non-overexpressed. For EGFR, the membrane immunostaining was scored following a 4-step scale (scores 0 , $1+, 2+$ and $3+)$. Slides with a score of $2+$ and $3+$ were classified as positive or overexpressed, in contrast to slides with a score of 0 or $1+$, which were defined as negative or non-overexpressed.

Cell culture. The CCA rat brain endothelial (RBE) and QBC939 cell lines were purchased from the Cell Bank of the Chinese Academy of Sciences (Shanghai, China). The CCA HUCCT1 cell line was obtained from RIKEN BioResource Center (Tsukuba, Japan). All lines were cultured in RPMI-1640 medium (Hyclone; GE Healthcare Life Sciences, Logan, UT, USA) supplemented with $10 \%$ fetal bovine serum (Gibco; Thermo Fisher Scientific, Inc., Waltham, MA, USA). The cells were maintained at $37^{\circ} \mathrm{C}$ in a humidified atmosphere with $5 \% \mathrm{CO}_{2}$.

In vitro overexpression of Cul4B. Human plasmids expressing Flag-tagged Cul4B (Flag-Cul4B) and negative control vector have been described previously (11). Cul4B and empty control plasmids were independently transfected into RBE cells using Lipofectamine 2000 (Invitrogen; Thermo Fisher Scientific, Inc.) according to the protocol of the manufacturer.
Small interfering RNA (siRNA)-mediated Cul4B knockdown. Three Cul4B-specific siRNAs were designed and synthesized by Shanghai GenePharma Co., Ltd. (Shanghai, China), and the most effective type of single siRNA (forward, 5'-GGC AGCACUAUUGUA AUUATT-3' and reverse, 5'-UAA UUACAAUAGUGCUGCCT-3') was used in additional experiments. A non-specific negative control siRNA was also designed and synthesized (forward, 5'- UUCUCCGAACGU GUCACGUTT-3' and reverse, 5'- ACGUGACACGUUCGG AGAATT-3').

Cell proliferation, migration and invasion assays. Cell proliferation was measured using a 3-(4,5-dimethylthiazol-2-yl)-5 -(3-carboxymethoxyphenyl)-2-(4-sulfophenyl)-2H-tetrazolium (MTS) assay with CellTiter $96^{\circledR} \mathrm{AQ}_{\text {ueous }}$ One Solution Reagent (Promega GmbH, Mannheim, Germany) following the protocol of the manufacturer. Migration and invasion assays were performed as previously described (15). To quantify the number of invading cells, the cells were counted in five randomly selected microscopic fields (magnification, $\mathrm{x} 200$ ). A total of three independent experiments were performed.

Soft agar colony assay. Soft agar colony assay was performed as previously described (16). HUCCT1 cells transfected with the indicated siRNAs or Flag-Cul4B were trypsinized and suspended in RPMI-1640 medium containing $0.3 \%$ lukewarm agar at a cell concentration of $5 \times 10^{3}$ cells $/ \mathrm{ml}$. The suspension was spread on top of $0.5 \%$ solidified agar plates. Colony formation was observed subsequent to a 2 -week culture. The colonies were counted and photographed using a CKX41 microscope (Olympus Corporation, Tokyo, Japan).

Reverse transcription-quantitative polymerase chain reaction $(R T-q P C R)$. Total RNA was extracted from the CCA cell lines using TRIzol (cat no. 15596026; Thermo Fisher Scientific, Inc.) and complementary DNA was synthesized by RT. A SYBR Green ${ }^{\circledR}$ Real-Time PCR Master Mix (Toyobo Co., Ltd., Osaka, Japan) and an ABI PRISM ${ }^{\circledR} 7700$ Sequence Detection System (Applied Biosystems; Thermo Fisher Scientific, Inc.) were used in the present study. The following thermocycling conditions were maintained: $50^{\circ} \mathrm{C}$ for $2 \mathrm{~min} ; 95^{\circ} \mathrm{C}$ for $10 \mathrm{~min}$; and 40 cycles of $95^{\circ} \mathrm{C}$ for $15 \mathrm{sec}$ and $60^{\circ} \mathrm{C}$ for $1 \mathrm{~min}$. The primers for Cul4B were as follows: Forward, 5'-TGGAAGTTC ATTTACCACCAGAGATG-3', and reverse, 5'-TTCTGCTTT TAACACACAGTGTCCTA-3'. The relative Cul4B expression was normalized to the messenger RNA (mRNA) expression of GAPDH, which was amplified with the following primers: Forward, 5'-GAGTCAACGGATTTGGTCGT-3', and reverse, 5'-TTGATTTTGGAGGGATCTC-3'. PCR assays were performed in triplicate, and fold induction was calculated using the $2^{-\Delta \Delta \mathrm{Cq}}$ method (17).

Western blot analysis. Western blot analysis was performed as previously described (15). Briefly, total protein was extracted using RIPA buffer (cat. no. 20-188; EMD Millipore, Billerica, MA, USA) and measured using a bicinchoninic acid protein assay kit (Beyotime Institute of Biotechnology, Haimen, China). Protein (20 $\mu \mathrm{g} /$ lane $)$ was directly electrophoresed using $10 \%$ SDS-PAGE and then transferred to an Immobilon-P 
Table I. Summary of demographics of patients with cholangiocarcinoma.

\begin{tabular}{lcr}
\hline Parameters & IHCC, n $\%)$ & EHCC, n $(\%)$ \\
\hline Age, years & & \\
$<60$ & $49(62.0)$ & $76(54.3)$ \\
$\geq 60$ & $30(38.0)$ & $64(45.7)$ \\
Gender & & \\
Male & $40(50.6)$ & $93(66.4)$ \\
Female & $39(49.4)$ & $47(33.6)$ \\
Tumor size, cm & & \\
$<5$ & $27(34.2)$ & $79(58.5)$ \\
$\geq 5$ & $52(65.8)$ & $56(41.5)$ \\
Histological differentiation & & $122(87.1)$ \\
Well and moderate & $60(75.9)$ & $18(12.9)$ \\
Poorly erately & $19(24.1)$ & \\
T stage & & $63(45.0)$ \\
I+II & $58(73.4)$ & $77(55.0)$ \\
III+IV & $21(26.6)$ & $104(74.3)$ \\
N stage & & $36(25.7)$ \\
Negative & $56(70.9)$ & \\
Positive & $23(29.1)$ & \\
UICC stage & & \\
I+II & & \\
III+IV & $36(54.4)$ & \\
\hline
\end{tabular}

UICC, Union for International Cancer Control; IHCC, intrahepatic cholangiocarcinoma; EHCC, extrahepatic cholangiocarcinoma.

polyvinylidene difluoride membrane (EMD Millipore, Billerica, MA, USA). The membranes were blocked at room temperature for $1 \mathrm{~h}$ in blocking buffer containing 3\% bovine serum albumin (Sigma-Aldrich; Merck KGaA), then incubated overnight at $4^{\circ} \mathrm{C}$ with the primary antibody against $\mathrm{Cul} 4 \mathrm{~B}$ (1:1,000; Sigma-Aldrich; Merck KGaA). Membranes were subsequently incubated with a horseradish peroxidase-conjugated anti-rabbit IgG secondary antibody (cat. no. 7074, 1:1500, Cell Signaling Technology, Inc., Danvers, MA, USA) at $37^{\circ} \mathrm{C}$ for $30 \mathrm{~min}$. Primary antibody directed against $\beta$-actin was used as a loading control (cat. no. sc-47778;, 1:5,000; Santa Cruz Biotechnology, Inc., Dallas, TX, USA). The signals were detected with an enhanced chemiluminescence reagent (Merck $\mathrm{KGaA}$ ). Three independent experiments were performed.

Statistical analysis. The software used for statistical analyses was SPSS version 19.0 (IBM SPSS, Armonk, NY, USA). Two-sided Student's t test and Mann-Whitney U test were used for statistical comparisons, while Spearman's rank correlation test was used to evaluate the correlations between Cul4B overexpression and a number of clinicopathological parameters. The Kaplan-Meier method and Cox regression hazard tests were applied for the analysis of follow-up data, and hazard ratio (HR) with $95 \%$ confidence intervals (CI) were calculated. $\mathrm{P}<0.05$ was considered to indicate a statistically significant difference.

\section{Results}

Expression of Cul4B and associations between Cul4B expression and clinicopathological factors in CCA. The present study performed IHC staining of Cul4B in a cohort of 219 patients with CCA to define the role of $\mathrm{Cul} 4 \mathrm{~B}$ in the progression of CCA. Predominantly nuclear staining of Cul4B was observed. $\mathrm{Cul} 4 \mathrm{~B}$ protein levels were greater in the cancer cells than in the adjacent benign ductal epithelia (data not shown). Representative images of Cul4B expression are shown in Fig. 1. Overall, Cul4B was overexpressed in $28.6 \%$ (40/140) of the patients with EHCC and in $26.6 \%(21 / 79)$ of the patients with IHCC. The associations between Cul4B overexpression and various clinicopathological factors are summarized in Table II. Among the patients with EHCC, Cul4B overexpression was significantly associated with higher pathological tumor stage $(\mathrm{P}=0.024)$ and lymph node metastasis $(\mathrm{P}=0.013)$. No associations were identified between $\mathrm{Cul} 4 \mathrm{~B}$ overexpression and gender $(\mathrm{P}=0.821)$, age $(\mathrm{P}=0.520)$, tumor size $(\mathrm{P}=0.650)$ or histological differentiation $(\mathrm{P}=0.299)$. By contrast, as shown in Table II, Cul4B expression was not associated with any clinicopathological variables in patients with IHCC. The overexpression of EGFR was present in $19(25.0 \%)$ of the 76 patients with IHCC and in $23(16.7 \%)$ of the 138 patients with EHCC. Notably, there was a marginal association between Cul4B overexpression and EGFR expression $(\mathrm{P}=0.093)$ in patients with $\mathrm{EHCC}$, but not in patients with IHCC.

Prognostic role of $C U l 4 B$ expression in $C C A$. To assess the possible association between Cul4B expression and patient survival, Kaplan-Meier curves with a log-rank test for overall survival (OS) were performed. In EHCC, as shown in Fig. 2, patients with Cul4B overexpression had a lower OS rate than patients who did not exhibit overexpression. The estimated mean OS time was significantly different between patients with Cul4B-overexpressed and patients with Cul4B-non-overexpressed tumors $(55.029 \pm 2.595$ and $86.974 \pm 0.882$ months, respectively; $\mathrm{P}<0.001)$. By contrast, no statistical significance $(\mathrm{P}=0.658)$ was identified between $\mathrm{Cul} 4 \mathrm{~B}$ overexpression and OS in IHCC.

In univariate Cox regression analysis, Cul4B overexpression was a prognostic factor for cancer mortality $(\mathrm{HR}=1.779$, 95\% CI=1.102-2.690, P=0.028; Table III) in EHCC. Additionally, histological differentiation $(\mathrm{P}=0.037)$, tumor stage $(\mathrm{P}=0.023)$, Union for International Cancer Control (UICC) stage $(\mathrm{P}<0.010)$ and EGFR expression were also significantly associated with OS. The classification of tumor stage and UICC stage is based on the 7th edition of the UICC-American Joint Committee on Cancer (UICC/AJCC) (18). In a multivariate analysis, UICC stage and EGFR expression exhibited predictive value, whereas Cul4B expression did not (Table III). In IHCC, Cul4B expression was not observed to be associated with the OS $(\mathrm{P}=0.768)$ of patients with CCA. Four factors, including tumor size, lymph node metastasis, UICC stage and EGFR expression, were identified as prognostic factors by univariate analysis. In the multivariate analysis, as shown in Table IV, only UICC stage and lymph node metastasis were independent prognostic factors. These results suggested that Cul4B was an unfavorable prognostic indicator in Chinese patients with EHCC. 

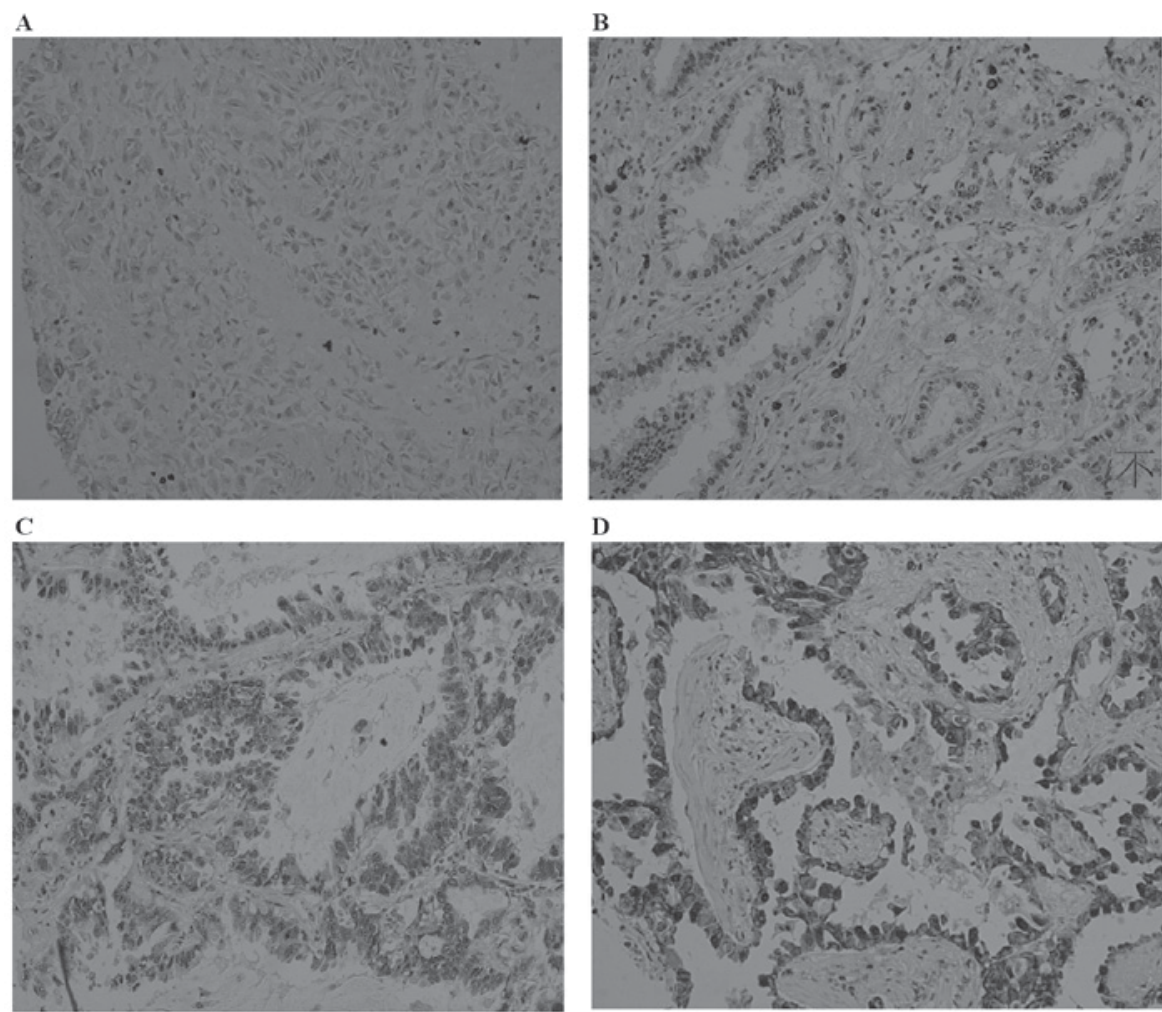

Figure 1. Expression of Cu14B protein in Chinese patients with cholangiocarcinoma by immunohistochemistry. (A) Negative staining, magnification, x200 (B) Weak staining, magnification, x200. (C) Moderate staining, magnification, x200. (D) Strong staining, magnification, x200.

A

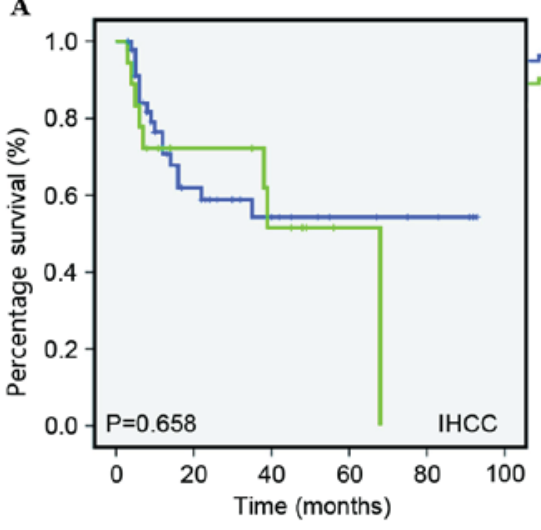

C

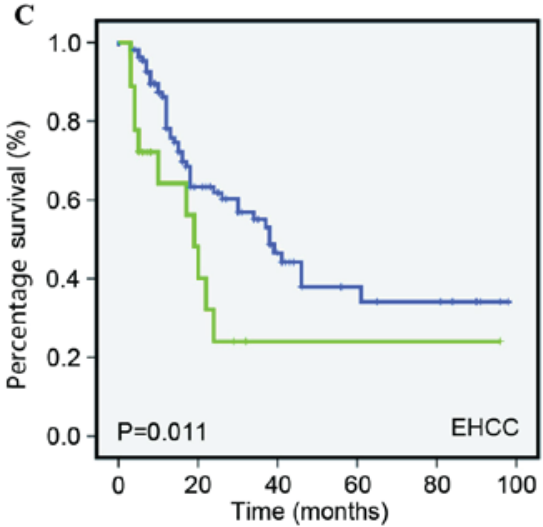

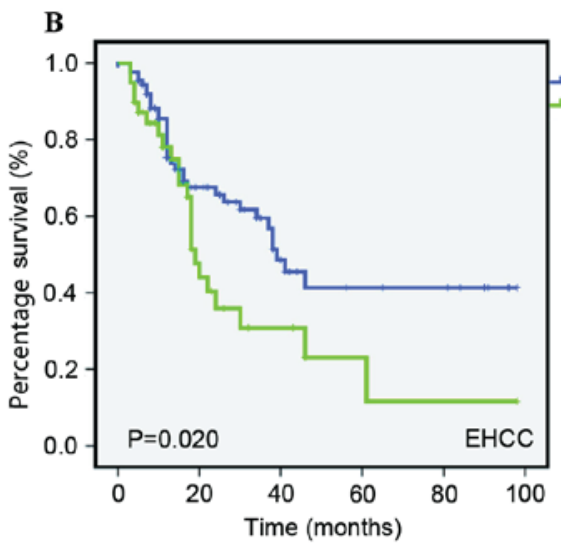

ras

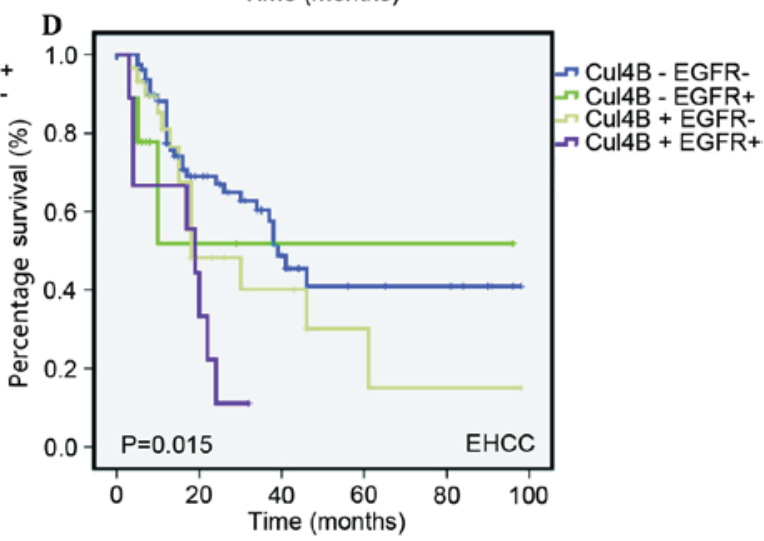

Figure 2. Kaplan-Meier survival analysis of Cul4B and EGFR expression in patients with cholangiocarcinoma. Survival curves stratified by Cul4B expression in (A) IHCC and (B) EHCC patients, respectively. (C) Survival curves stratified by EGFR expression in EHCC patients. (D) Survival curves stratified by Cul4B and EGFR expression in combination. Cancer-associated mortality was used as the end point. IHCC, intrahepatic cholangiocarcinoma; EHCC, extrahepatic cholangiocarcinoma; Cul, cullin; EGFR, epidermal growth factor receptor. 
Table II. Association of Cul4B expression level with clinicopathological parameters in cholangiocarcinoma.

\begin{tabular}{|c|c|c|c|c|c|c|}
\hline \multirow[b]{2}{*}{ Parameters } & \multicolumn{2}{|c|}{ Cul4B in IHCC } & \multirow[b]{2}{*}{ P-value } & \multicolumn{2}{|c|}{ Cul4B in EHCC } & \multirow[b]{2}{*}{ P-value } \\
\hline & $\begin{array}{c}\text { Not } \\
\text { overexpressed, } \\
\mathrm{n}(\%)\end{array}$ & $\begin{array}{c}\text { Over } \\
\text { expressed, } \\
\text { n }(\%)\end{array}$ & & $\begin{array}{c}\text { Not } \\
\text { overexpressed, } \\
\mathrm{n}(\%)\end{array}$ & $\begin{array}{c}\text { Over } \\
\text { expressed, } \\
\text { n (\%) }\end{array}$ & \\
\hline Age, years & & & 0.989 & & & 0.520 \\
\hline$<60$ & $36(73.5)$ & $13(26.5)$ & & $56(71.8)$ & $20(28.2)$ & \\
\hline$\geq 60$ & $22(73.3)$ & $8(26.7)$ & & $44(68.8)$ & $20(31.2)$ & \\
\hline Gender & & & 0.486 & & & 0.821 \\
\hline Male & $28(70.0)$ & $12(30.0)$ & & $67(72.0)$ & $26(28.0)$ & \\
\hline Female & $30(76.9)$ & $9(23.1)$ & & $33(70.3)$ & $14(29.7)$ & \\
\hline Tumor size, $\mathrm{cm}$ & & & 0.328 & & & 0.650 \\
\hline$<5$ & $18(66.7)$ & $9(33.3)$ & & $55(69.6)$ & $24(30.4)$ & \\
\hline$\geq 5$ & $40(76.9)$ & $12(23.1)$ & & $41(73.2)$ & $15(26.8)$ & \\
\hline Histological differentiation & & & 0.531 & & & 0.299 \\
\hline Well and moderate & $43(71.7)$ & $17(28.3)$ & & & & \\
\hline Poor & $15(78.9)$ & $4(21.1)$ & & $11(61.1)$ & $7(38.9)$ & \\
\hline T stage & & & 0.630 & & & 0.024 \\
\hline $\mathrm{I}+\mathrm{II}$ & $41(71.9)$ & $16(28.1)$ & & $39(61.9)$ & $24(38.1)$ & \\
\hline $\mathrm{III}+\mathrm{IV}$ & $17(77.3)$ & $5(22.7)$ & & $61(79.2)$ & $16(20.8)$ & \\
\hline Lymph node metastasis & & & 0.422 & & & 0.013 \\
\hline Negative & $42(75.0)$ & $14(25.0)$ & & $68(65.4)$ & $36(34.6)$ & \\
\hline Positive & $16(69.6)$ & $7(30.4)$ & & $32(88.8)$ & $4(11.2)$ & \\
\hline UICC stage & & & 0.619 & & & 0.217 \\
\hline $\mathrm{I}+\mathrm{II}$ & $30(69.8)$ & $13(30.2)$ & & $51(67.1)$ & $25(32.9)$ & \\
\hline $\mathrm{III}+\mathrm{IV}$ & $28(77.8)$ & $8(22.2)$ & & 49 (76.6) & $15(23.4)$ & \\
\hline EGFR expression & & & 0.139 & & & 0.093 \\
\hline Not overexpressed & $44(77.2)$ & $13(22.8)$ & & $85(73.9)$ & $30(26.1)$ & \\
\hline Overexpressed & $11(57.9)$ & $8(42.1)$ & & $13(56.5)$ & $10(43.5)$ & \\
\hline
\end{tabular}

IHCC, intrahepatic cholangiocarcinoma; EHCC, extrahepatic cholangiocarcinoma; UICC, Union for International Cancer Control; EGFR, epidermal growth factor receptor.

Cul4B+/EGFR+ defines a subset of EHCC patients with poor prognosis. The present study then determined whether combining Cu14B and EGFR further improved their prognostic value in patients with EHCC, by grouping all patients with EHCC according to Cul4B and EGFR overexpression status. Kaplan-Meier analyses were then conducted using the group exhibiting neither Cul4B overexpression nor EGFR overexpression as the reference. As shown in Fig. 2D, the group possessing EGFR and Cul4B overexpression exhibited the worst cancer-associated survival compared with the three other groups.

Cul4B promotes proliferation, migration and invasion in CCA cells. To explore the biological role of Cul4B in CCA in vitro, the present study first evaluated the endogenous expression of Cul4B in different CCA cell lines. As shown in Fig. 3A, QBC939 cells exhibited the highest level of Cul4B, whereas HUCCT1 and RBE cells exhibited relatively low levels of Cul4B (QBC939>HUCCT1>RBE). Using an
MTS assay, the present study revealed that the proliferation of QBC939 cells was significantly reduced subsequent to the silencing of Cul4B compared with that of the negative control ( $\mathrm{P}=0.033$; Fig. 3B). Subsequent to 48 and $72 \mathrm{~h}$ of Cul4B siRNA treatment, the number of QBC939 cells was reduced by $20.7 \pm 3.5$ and $27.9 \pm 5.6 \%$, respectively (Fig. 3B). In addition, the present study also performed overexpression of Cul4B in RBE cells. The growth of RBE cells increased significantly subsequent to transfection with a Cul4B expression plasmid ( 48 h, $\mathrm{P}=0.034 ; 96$ h, $\mathrm{P}=0.024$; Fig. 3B). The overexpression of Cul4B significantly increased the level of colony formation of HUCCT1 cells compared with that of the negative control ( $\mathrm{P}=0.006$; Fig. $3 \mathrm{C})$. Transwell experiments were then performed to determine the migration and invasive abilities of CCA cells either transfected with siRNA or with the Cul4B expression plasmid. The data of the present study revealed that the migration and invasive capacities of QBC939 and HUCCT1 cells decreased subsequent to the knocking down of Cul4B. By contrast, overexpression of 
Table III. Univariate and multivariate analysis for overall survival in extrahepatic cholangiocarcinoma.

\begin{tabular}{|c|c|c|c|c|c|c|}
\hline \multirow[b]{2}{*}{ Parameters } & \multicolumn{3}{|c|}{ Univariate analysis } & \multicolumn{3}{|c|}{ Multivariate analysis } \\
\hline & HR & $95 \% \mathrm{CI}$ & P-value & HR & $95 \% \mathrm{CI}$ & P-value \\
\hline \multicolumn{7}{|l|}{ Age, years } \\
\hline$<60$ & 1 & (Reference) & & 1 & (Reference) & \\
\hline$\geq 60$ & 1.533 & $0.947-2.481$ & 0.082 & - & - & - \\
\hline \multicolumn{7}{|l|}{ Gender } \\
\hline Male & 1 & (Reference) & & 1 & (Reference) & \\
\hline Female & 0.831 & $0.496-1.394$ & 0.484 & - & - & - \\
\hline \multicolumn{7}{|l|}{ Tumor size, $\mathrm{cm}$} \\
\hline$<3$ & 1 & (Reference) & & 1 & (Reference) & \\
\hline$\geq 3$ & 1.06 & $0.642-1.749$ & 0.820 & - & - & - \\
\hline \multicolumn{7}{|c|}{ Histological differentiation } \\
\hline Well and moderate & 1 & (Reference) & & 1 & (Reference) & \\
\hline Poor & 0.54 & 0.303-0.962 & 0.037 & 0.920 & $0.478-1.796$ & 0.821 \\
\hline \multicolumn{7}{|l|}{ T stage } \\
\hline $\mathrm{I}+\mathrm{II}$ & 1 & (Reference) & & 1 & (Reference) & \\
\hline III+IV & 1.782 & $1.084-2.929$ & 0.023 & 1.199 & $0.703-2.045$ & 0.506 \\
\hline \multicolumn{7}{|c|}{ Lymph node metastasis } \\
\hline Negative & 1 & (Reference) & & 1 & (Reference) & \\
\hline Positive & 1.288 & $0.734-2.263$ & 0.378 & & & \\
\hline \multicolumn{7}{|l|}{ UICC stage } \\
\hline $\mathrm{I}+\mathrm{II}$ & 1 & (Reference) & & 1 & (Reference) & \\
\hline III+IV & 3.141 & $1.931-5.110$ & $<0.010$ & 3.083 & $1.894-5.018$ & $<0.010$ \\
\hline \multicolumn{7}{|l|}{ Cul4B expression } \\
\hline Not overexpressed & 1 & (Reference) & & 1 & (Reference) & \\
\hline Overexpressed & 1.779 & $1.102-2.690$ & 0.028 & 1.358 & $0.809-2.423$ & 0.162 \\
\hline \multicolumn{7}{|l|}{ EGFR expression } \\
\hline Not overexpressed & 1 & (Reference) & & 1 & (Reference) & \\
\hline Overexpressed & 1.989 & $1.100-3.600$ & 0.023 & 1.876 & $1.038-3.390$ & 0.037 \\
\hline
\end{tabular}

HR, hazard ratio; CI, confidence interval; UICC, Union for International Cancer Control; EGFR, epidermal growth factor receptor; Cul, cullin.

Cul4B significantly increased the migration and invasive capacities of HUCCT1and RBE cells (HUCCT1, $\mathrm{P}=0.014$; RBE, P=0.017; Fig. 3D).

In vitro effect of Cul4B on EMT. The present study subsequently investigated whether $\mathrm{Cul} 4 \mathrm{~B}$ is a regulator of EMT in CCA in vitro. Of note, Cul4B overexpression induced an elongated fibroblast-like morphology with scattered distribution in cultured RBE cells (Fig. 4A). Cul4B was then transiently knocked down, resulting in the suppression of the EMT of CCA cells, as shown by the inhibition of E-cadherin expression (an epithelial marker) and an increase in vimentin and $\mathrm{N}$-cadherin (mesenchymal markers) at the protein level (Fig. 4B).

Modulation of p16 and phosphatase and tensin homolog (PTEN) by Cul4B in CCA cell lines. Hu et al (11) reported that $\mathrm{Cul} 4 \mathrm{~B}$ may contribute to the transcriptional regulation of tumor-suppressor genes in human breast adenocarcinoma cell lines. The present study therefore investigated the modulation of two known tumor suppressor genes, P16 and PTEN, by Cul4B. As shown in Fig. 4C, siRNA knockdown of Cul4B led to a significant upregulation of p16 and PTEN expression at the mRNA level in QBC939 cells (P16, $\mathrm{P}=0.049$; PTEN, $\mathrm{P}=0.041$ ), suggesting that $\mathrm{Cul}$ B $\mathrm{B}$ promotes tumor progression partially through the repression of the aforementioned tumor-suppressor genes.

\section{Discussion}

Cul4B is a scaffold protein of the CRL complex, and is involved in the regulation of a broad spectrum of biological processes, including cell cycle progression, DNA replication and DNA damage response $(6,7,19)$. Cul4B has previously been shown to be overexpressed in various types of solid malignancy $(9-11,16,19,20)$. However, the precise role of Cul4B in CCA is unknown. The present study provides support for the hypothesis that $\mathrm{Cul} 4 \mathrm{~B}$ serves an oncogenic role in CCA 
Table IV. Univariate and multivariate analysis for overall survival in intrahepatic cholangiocarcinoma.

\begin{tabular}{|c|c|c|c|c|c|c|}
\hline \multirow[b]{2}{*}{ Parameters } & \multicolumn{3}{|c|}{ Univariate analysis } & \multicolumn{3}{|c|}{ Multivariate analysis } \\
\hline & $\mathrm{HR}$ & $95 \% \mathrm{CI}$ & P-value & HR & $95 \% \mathrm{CI}$ & P-value \\
\hline \multicolumn{7}{|l|}{ Age, years } \\
\hline$<60$ & 1 & (Reference) & & 1 & (Reference) & \\
\hline$\geq 60$ & 1.003 & $0.518-1.941$ & 0.993 & - & - & - \\
\hline \multicolumn{7}{|l|}{ Gender } \\
\hline Male & 1 & (Reference) & & 1 & (Reference) & \\
\hline Female & 0.555 & $0.289-1.067$ & 0.177 & - & - & - \\
\hline \multicolumn{7}{|l|}{ Tumor size, cm } \\
\hline$<5$ & 1 & (Reference) & & 1 & (Reference) & \\
\hline$\geq 5$ & 2.136 & $1.006-4.536$ & 0.048 & 1.711 & $0.778-3.764$ & 0.181 \\
\hline \multicolumn{7}{|c|}{ Histological differentiation } \\
\hline Well and moderate & 1 & (Reference) & & 1 & (Reference) & \\
\hline Poor & 0.558 & $0.285-1.094$ & 0.089 & - & - & - \\
\hline \multicolumn{7}{|l|}{ T stage } \\
\hline I+II & 1 & (Reference) & & 1 & (Reference) & \\
\hline III+IV & 1.532 & $0.791-2.968$ & 0.206 & - & - & - \\
\hline \multicolumn{7}{|c|}{ Lymph node metastasis } \\
\hline Negative & 1 & (Reference) & & 1 & (Reference) & \\
\hline Positive & 4.362 & $2.210-8.610$ & $<0.010$ & 2.402 & $1.065-5.418$ & 0.035 \\
\hline \multicolumn{7}{|l|}{ UICC stage } \\
\hline I+II & 1 & (Reference) & & 1 & (Reference) & \\
\hline III+IV & 3.935 & $1.969-7.867$ & $<0.010$ & 2.566 & $1.110-5.935$ & 0.028 \\
\hline \multicolumn{7}{|l|}{ Cul4B expression } \\
\hline Not overexpressed & 1 & (Reference) & & 1 & (Reference) & \\
\hline Overexpressed & 1.112 & $0.550-2.246$ & 0.768 & - & - & - \\
\hline \multicolumn{7}{|l|}{ EGFR expression } \\
\hline Not overexpressed & 1 & (Reference) & & 1 & (Reference) & \\
\hline Overexpressed & 1.866 & $1.215-3.505$ & 0.030 & 1.453 & $1.206-2.815$ & 0.285 \\
\hline
\end{tabular}

HR, hazard ratio; CI, confidence interval; UICC, Union for International Cancer Control; EGFR, epidermal growth factor receptor; Cul, cullin.

progression. Firstly, the data of the present study clearly demonstrated that siRNA knockdown of Cul4B significantly inhibits the proliferation and soft agar growth of CCA cells in vitro. Secondly, siRNA knockdown of Cul4B significantly decreased the migration and invasive capacities of CCA cells, which are two critical events in the progression of cancer towards metastasis. Therefore, Cul4B overexpression is significantly associated with the presence of lymph node metastasis and higher clinical tumor stages in clinical CCA cases. Thirdly $\mathrm{Cul}$ B is an unfavorable prognostic factor in a subset of patients with EHCC. In concordance with these findings, Jiang et al (9) reported that high $\mathrm{Cul}$ B B expression was associated with the depth of tumor invasion, lymph node metastasis, distant metastasis, histological differentiation, vascular invasion and advanced tumor stage of numerous types of colon cancer.

The overexpression of Cul4B in a subset of patients with CCA may result from the activation of Cul4B by increased transcription, possibly through amplification or promoter mutations. Alternatively, Cul4B may be activated by an unknown upstream genetic event. It has been reported that $\mathrm{Cul} 4 \mathrm{~A}$, another member of CRL4, was amplified in primary breast cancer, squamous cell carcinoma, adrenocortical carcinoma and hepatocellular carcinoma (19). Previously, genome-wide high-density single nucleotide polymorphism arrays further revealed a high Cul4A gene copy number in a subset of lung and ovarian carcinoma patients (21). Although mutations of the Cul4B gene are causally associated with human X-linked mental retardation (19), the data are limited regarding aberrations of Cul4B at the DNA level in cancer. Therefore, additional investigation into the genetic characterization of Cul4B in CCA using clinical samples is required.

The mechanism by which $\mathrm{Cul} 4 \mathrm{~B}$ contributes to cancer invasion and progression remains undefined. Cul4B may exert oncogenicity in several ways. Previously, $\mathrm{Hu}$ et al (11) demonstrated that, by catalyzing histone $(\mathrm{H}) 2$ AK119 monoubiquitination and coordinating with polycomb-repressive complex 2, Cul4B can promote the transcriptional silencing of an array of tumor-suppressor genes. Additionally, 
A
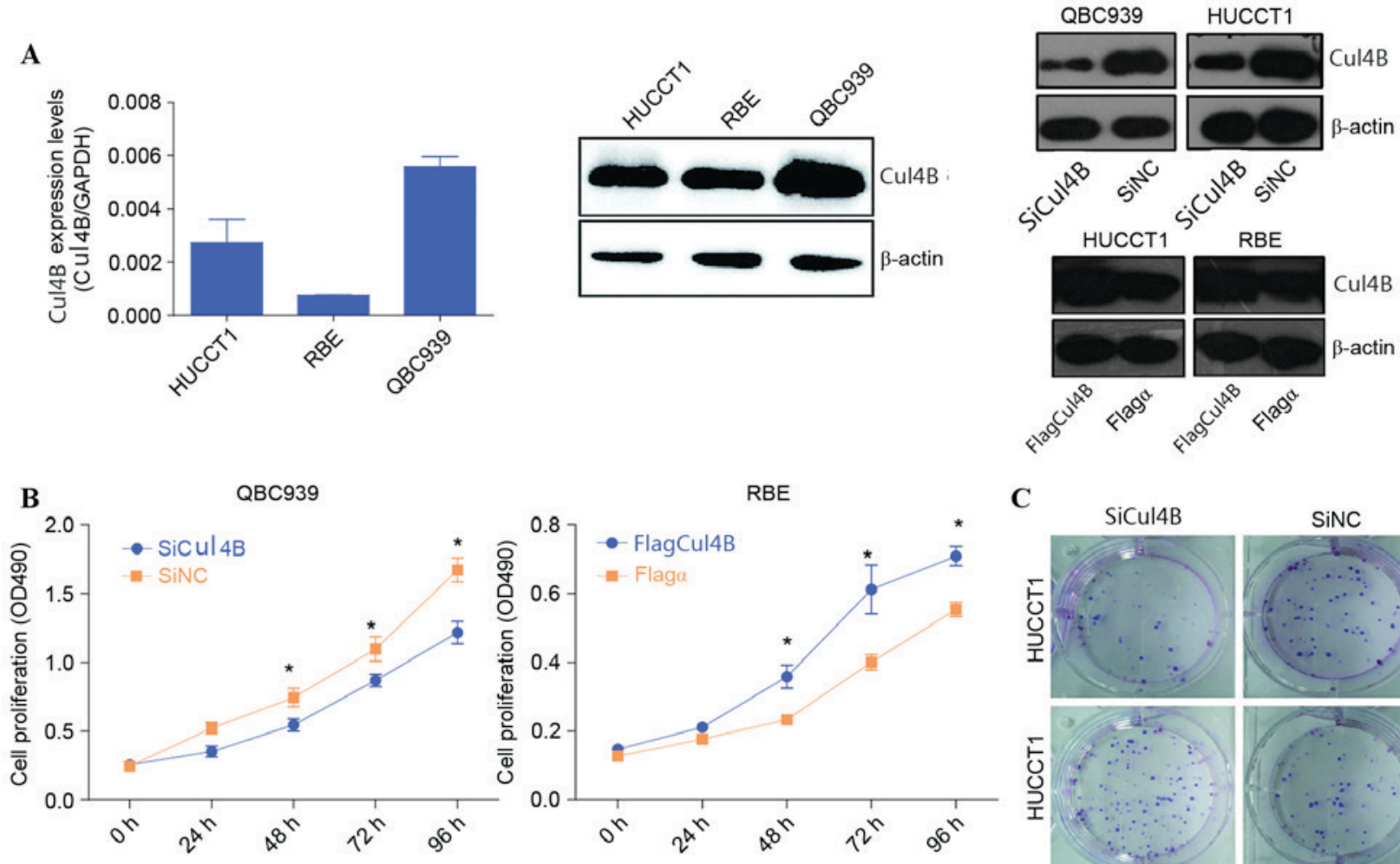

C
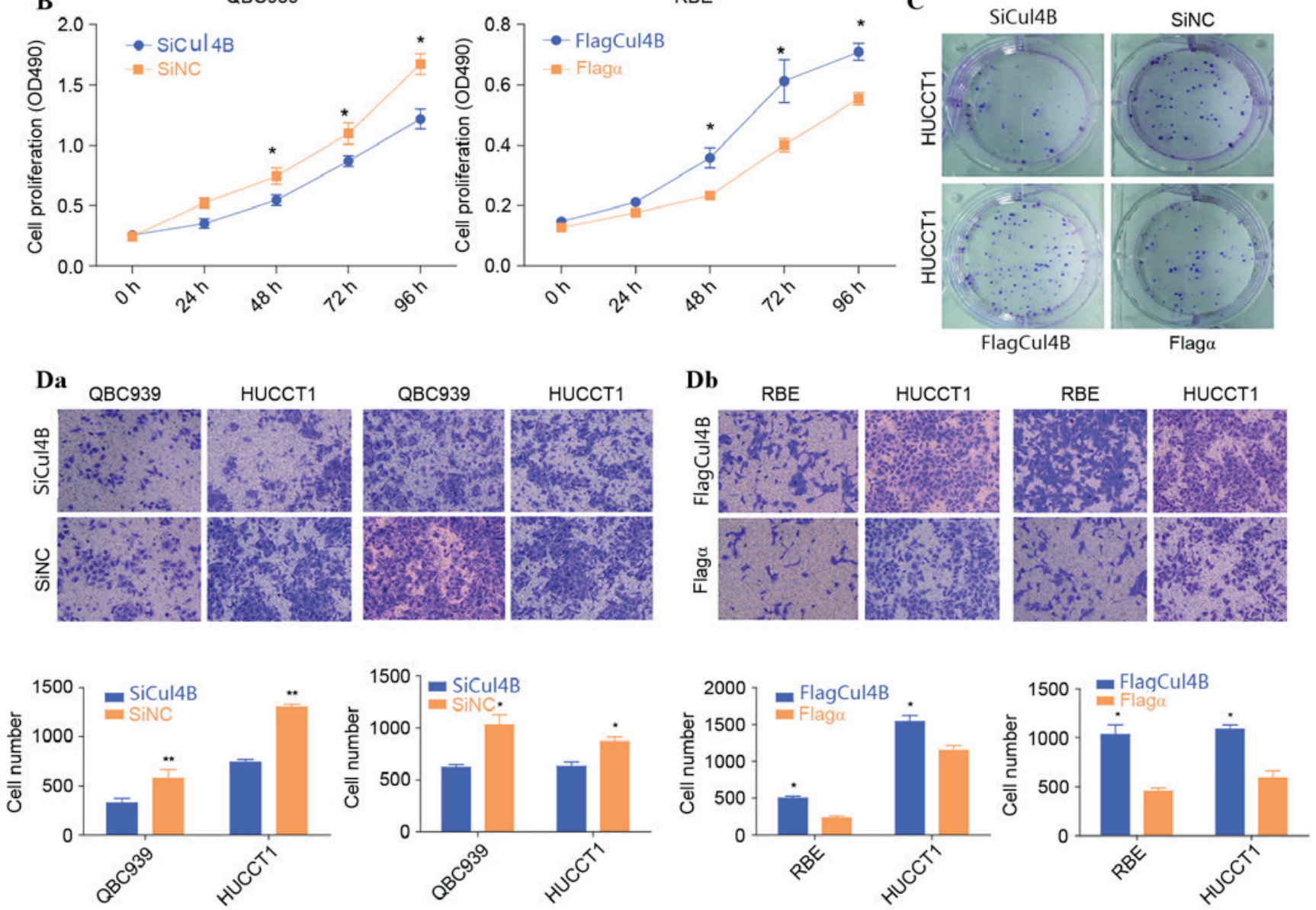

Figure 3. Biological roles of Cu14B on CCA in vitro. (A) The messenger RNA and protein levels of Cul4B in HUCCT1, RBE and QBC939 cell lines were determined by reverse transcription-quantitative polymerase chain reaction and western blot analysis, respectively. Protein expression levels of Cul4B following the overexpression and siRNA knockdown of the gene are also shown. $\beta$-Actin and GAPDH were used as internal references. (B) 3-(4,5-Dimethylthiazol-2-yl)5-(3-carboxymethoxyphenyl)-2-(4-sulfophenyl)-2H-tetrazolium assays were performed to examine growth rates at different time points ranging between 0 and $96 \mathrm{~h}$ in QBC939 and RBE cells. (C) A colony formation assay was performed to examine the colony formation ability of HUCCT1 cells following siRNA knockdown and overexpression of Cul4B. (Da) Cellular migration and (Db) invasion capacities of CCA cells were evaluated by Transwell assays. Representative images of migratory and invasive cells were shown. Statistical results of cell numbers at the bottom of the membrane were visualized and are presented as the mean \pm standard deviation. ${ }^{*} \mathrm{P}<0.05,{ }^{* *} \mathrm{P}<0.01$ vs. SiCul4B or Flag $\alpha$. SiCul4B, siRNA knockdown of Cul4B; SiNC, negative control siRNA; CCA, cholangiocarcinoma; RBE, rat brain endothelial; siRNA, small interfering RNA; Cul, cullin; OD, optical density.

Yang et al (16) reported that Cul4B promotes tumorigenesis by coordinating with suppressor of variegation 3-9 homolog 1/heterochromatin protein1/DNA (cytosine-5)-methyltransferase $3 \mathrm{~A}$ in DNA methylation-based epigenetic silencing. The depletion of Cul4B resulted in H3K9 trimethylation and DNA methylation, leading to the repression of a collection of genes, including the tumor suppressor insulin-like growth factor-binding protein 3 . Consistently with these findings, the present study noticed that siRNA knockdown of Cul4B significantly downregulated the expression of P16 and PTEN in CCA cells. It was recently reported that Cul4B upregulates Wnt/ $\beta$-catenin signaling in hepatocellular carcinoma (HCC) through transcriptionally repressing Wnt antagonists, thus contributing to the malignancy of HCC (10). 
Aa

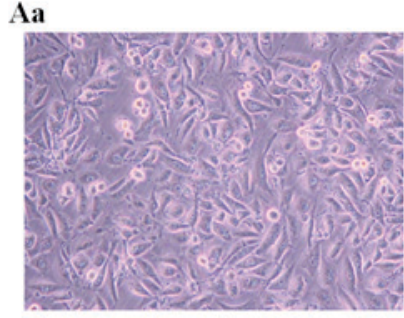

C

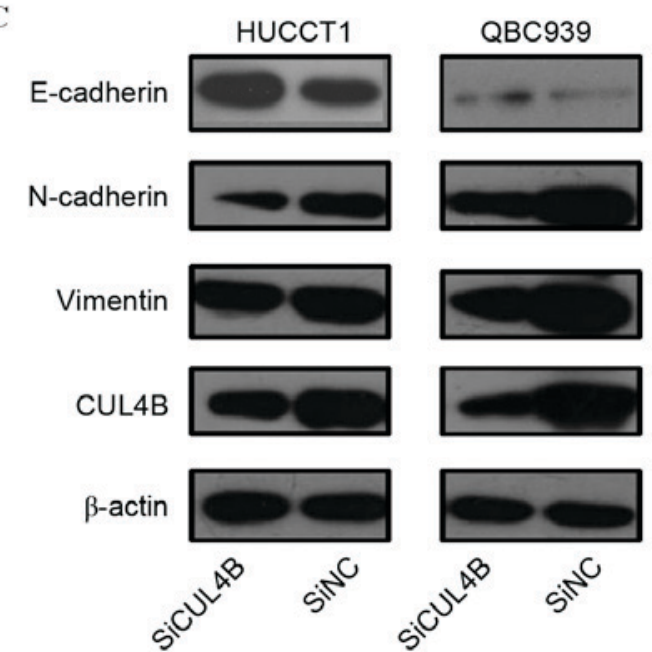

Ab

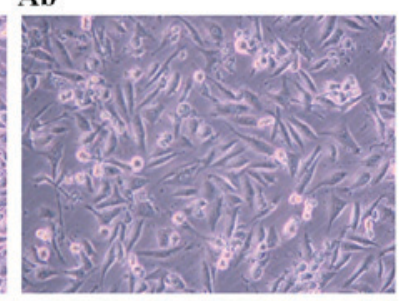

B
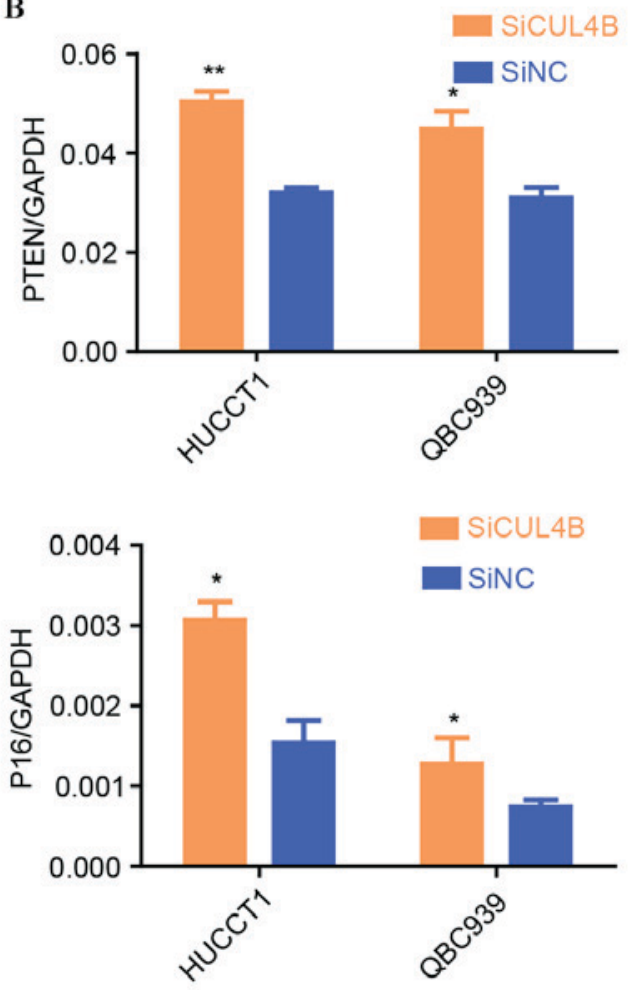

Figure 4. In vitro effect of Cul4B on EMT and modulation of P16 and PTEN by Cul4B. (A) Morphological changes of QBC939 cells with siRNA knockdown of Cul4B (magnification, x200) (Aa) prior to treatment and (Ab) subsequent to siRNA knockdown of Cul4B for 48 h. (B) Expression levels of PTEN and P16 as determined by reverse transcription-quantitative polymerase chain reaction subsequent to siRNA Cu14B transfection in HUCTT1 and QBC939 cells. Representative results from triplicate experiments are shown as the mean + standard deviation. ${ }^{*} \mathrm{P}<0.05,{ }^{* *} \mathrm{P}<0.01 \mathrm{vs}$. siNC. All target gene relative expression was normalized to GAPDH. (C) Protein expression levels of Cul4B, the mesenchymal markers vimentin and N-cadherin, and the epithelial marker E-cadherin are shown subsequent to siRNA Cul4B in HUCTT1 and QBC939 cells. SiCul4B, siRNA knockdown of Cul4B; SiNC, negative control siRNA; siRNA, small interfering RNA; Cul, cullin; PTEN, phosphatase and tensin homolog.

The present study demonstrated that $\mathrm{Cu}$ 14B promoted EMT of CCA cells. EMT is a developmental program, and is essential for tumor cells to disseminate to adjacent tissues and establish new tumors in distant sites (15). The data of the present study suggest that siRNA knockdown of Cul4B results in the upregulation of E-cadherin and the downregulation of vimentin in QBC939 and HUCCT1 cells, respectively. As EMT is an important process implicated in invasion and metastasis, these results may partially explain the mechanism by which Cul4B promotes CCA progression.

Clinically, the precise stratification of CCA patients according to their clinical prognosis in alignment with therapeutic options remains a challenge (3). A number of studies have identified multiple biomarkers that appear to possess prognostic significance. Of these, p53 mutation, cyclins, cancer antigen19-9 and connective tissue growth factor appeared to serve as predictors of outcome (5). The present study demonstrated that Cul4B expression is an unfavorable prognostic factor in Chinese patients with CCA. A negative correlation between Cul4B expression and OS in patients with EHCC, but not in patients with IHCC, was also revealed. The lack of association of Cul4B expression with poor survival in patients with IHCC may be attributed to the small number of patients, included in the study, or the fact that patients with CCA at different sites may present different pathogenic features. It has previously been reported that CCA differentially expresses cell cycle regulatory proteins based on tumor location and morphology (22).

The present study demonstrated that the co-overexpression of Cul4B and EGFR defines a subset of EHCC patients with poor prognosis. A marginally positive correlation between Cul4B and EGFR overexpression in EHCC patients was revealed. EGFR has been suggested to be an important prognostic factor and a potential therapeutic target in CCA (23). Wang et al (24) reported that Cul4A, another member of $\mathrm{Cul} 4$, may be a promising therapy target and a potential biomarker for prognosis and EGFR target therapy in patients with lung cancer. Therefore, the ability of Cul4B to regulate EGFR expression in CCA requires additional investigation.

In conclusion, the present study is the first to demonstrate that the overexpression of Cul4B is an unfavorable prognostic factor in Chinese patients with EHCC. Determining Cul4B overexpression in surgically excised CCA tissues may aid to predict the OS of patients. Additionally, these findings suggest that Cul4B and EGFR expression may define a subset of patients with CCA with poor prognosis.

\section{Acknowledgements}

The present study was supported by the National Natural Science Foundation of China (Beijing, China; grant nos., 81072110, 81171951, 81330050 and 81672554). 


\section{References}

1. Blechacz B, Komuta M, Roskams T and Gores GJ: Clinical diagnosis and staging of cholangiocarcinoma. Nat Rev Gastroenterol Hepatol 8: 512-522, 2011.

2. Patel T: Cholangiocarcinoma-controversies and challenges. Nat Rev Gastroenterol Hepatol 8: 189-200, 2011.

3. Malhi $\mathrm{H}$ and Gores GJ: Cholangiocarcinoma: Modern advances in understanding a deadly old disease. J Hepatol 45: 856-867, 2006.

4. Hezel AF and Zhu AX: Systemic therapy for biliary tract cancers. Oncologist 13: 415-423, 2008.

5. Briggs CD, Neal CP, Mann CD, Steward WP, Manson MM and Berry DP: Prognostic molecular markers in cholangiocarcinoma: A systematic review. Eur J Cancer 45: 33-47, 2009.

6. Petroski MD and Deshaies RJ: Function and regulation of cullin-RING ubiquitin ligases. Nat Rev Mol Cell Biol 6: 9-20, 2005

7. Sarikas A, Hartmann T and Pan ZQ: The cullin protein family. Genome Biol 12: 220, 2011.

8. Zou Y, Mi J, Cui J, Lu D, Zhang X, Guo C, Gao G, Liu Q, Chen B, Shao $\mathrm{C}$ and Gong Y: Characterization of nuclear localization signal in the $\mathrm{N}$ terminus of CUL4B and its essential role in cyclin E degradation and cell cycle progression. J Biol Chem 284: 33320-33332, 2009.

9. Jiang T, Tang HM, Wu ZH, Chen J, Lu S, Zhou CZ, Yan DW and Peng ZH: Cullin 4B is a novel prognostic marker that correlates with colon cancer progression and pathogenesis. Med Oncol 30: 534, 2013.

10. Yuan J, Han B, Hu H, Qian Y, Liu Z, Wei Z, Liang X, Jiang B, Shao $C$ and Gong Y: CUL4B activates Wnt/ $\beta$-catenin signalling in hepatocellular carcinoma by repressing Wnt antagonists. J Pathol 235: 784-795, 2015.

11. Hu H, Yang Y, Ji Q, Zhao W, Jiang B, Liu R, Yuan J, Liu Q, Li X, Zou Y, et al: CRL4B catalyzes H2AK119 monoubiquitination and coordinates with PRC2 to promote tumorigenesis. Cancer Cell 22: 781-795, 2012.

12. Nauseef JT and Henry MD: Epithelial-to-mesenchymal transition in prostate cancer: Paradigm or puzzle? Nat Rev Urol 8 : 428-439, 2011

13. Thompson EW, Newgreen DF and Tarin D: Carcinoma invasion and metastasis: A role for epithelial-mesenchymal transition? Cancer Res 65: 5991-5995, 2015.
14. Kong D, Banerjee S, Ahmad A, Li Y, Wang Z, Sethi S and Sarkar FH: Epithelial to mesenchymal transition is mechanistically linked with stem cell signatures in prostate cancer cells. PLoS One 5: e12445, 2010.

15. Wang L, Zhang J, Yang X, Chang YW, Qi M, Zhou Z, Zhang J and Han B: SOX4 is associated with poor prognosis in prostate cancer and promotes epithelial-mesenchymal transition in vitro. Prostate Cancer Prostatic Dis 16: 301-307, 2013.

16. Yang Y, Liu R, Qiu R, Zheng Y, Huang W, Hu H, Ji Q, He H, Shang Y, Gong Y and Wang Y: CRL4B promotes tumorigenesis by coordinating with SUV39H1/HP1/DNMT3A in DNA methylation-based epigenetic silencing. Oncogene 34: 104-118, 2015.

17. Livak KJ and Schmittgen TD: Analysis of relative gene expression data using real-time quantitative PCR and the 2(-Delta Delta C(T)) method. Method 25: 402-408, 2011.

18. Edge SB and Compton CC: The American Joint Committee on Cancer: The 7th edition of the AJCC cancer staging manual and the future of TNM. Ann Surg Oncol 17: 1471-1474, 2010.

19. Lee J and Zhou P: Pathogenic role of the CRL4 ubiquitin ligase in human disease. Front Oncol 2: 21, 2012.

20. Mok MT and Cheng AS: CUL4B: A novel epigenetic driver in Wnt/ $\beta$-catenin-dependent hepatocarcinogenesis. J Pathol 236: $1-4,2015$

21. Beroukhim R, Mermel CH, Porter D, Wei G, Raychaudhuri S, Donovan J, Barretina J, Boehm JS, Dobson J, Urashima M, et al: The landscape of somatic copy-number alteration across human cancers. Nature 463: 899-905, 2010.

22. Jarnagin WR, Klimstra DS, Hezel M, Gonen M, Fong Y, Roggin K, Cymes K, DeMatteo RP, D'Angelica M, Blumgart LH and Singh B: Differential cell cycle-regulatory protein expression in biliary tract adenocarcinoma: Correlation with anatomic site, pathologic variables, and clinical outcome. J Clin Oncol 24: 1152-1160, 2006

23. Zhang H, Berezov A, Wang Q, Zhang G, Drebin J, Murali R and Greene MI: ErbB receptors: From oncogenes to targeted cancer therapies. J Clin Invest 117: 2051-2058, 2007.

24. Wang Y, Zhang P, Liu Z, Wang Q, Wen M, Wang Y, Yuan H, Mao JH and Wei G: CUL4A overexpression enhances lung tumor growth and sensitizes lung cancer cells to erlotinib via transcriptional regulation of EGFR. Mol Cancer 13: 252, 2014. 